\title{
Multistability of a two-component exciton-polariton fluid
}

\author{
E. Cancellieri, ${ }^{1, *}$ F. M. Marchetti, ${ }^{1}$ M. H. Szymańska, ${ }^{2,3}$ and C. Tejedor ${ }^{1}$ \\ ${ }^{1}$ Física Teórica de la Materia Condensada, Universidad Autónoma de Madrid, Madrid ES-28049, Spain \\ ${ }^{2}$ Department of Physics, University of Warwick, Coventry, United Kindom \\ ${ }^{3}$ London Centre for Nanotechnology, United Kindom
}

(Received 10 January 2011; revised manuscript received 8 April 2011; published 6 June 2011)

\begin{abstract}
We study the stability of a multicomponent exciton-polariton fluid under resonant excitation within the linearresponse approximation of a generalized Gross-Pitaevskii equation. We show that two spatially homogeneous and independently tunable pumping lasers produce, for the same values of the system parameters, up to three stable solutions. Tristability is understood by noting that the cavity can be either lowly or highly populated and, in this second case, the largest part of the population lies in either one of the two components. Moreover, we discuss the different kinds of instabilities appearing at different pump intensities and compare them with the case of one-component fluids. Finally, we show that easily tunable multistable hysteresis loops can be performed by the system.
\end{abstract}

DOI: 10.1103/PhysRevB.83.214507

PACS number(s): 71.36.+c, 42.65.Pc, 03.75.Kk

\section{INTRODUCTION}

Condensates of resonantly pumped exciton polaritons in semiconductor microcavities constitute a novel and exciting system for the study of fundamental physical properties of superfluids out of equilibrium, ${ }^{1}$ and for future device applications. ${ }^{2,3}$ Being quantum superpositions of light and matter they are privileged candidates for the realization of the next generation of optical devices, ${ }^{2}$ for example, for quantum information technologies.

From the point of view of the fundamental physical properties, particularly interesting is the case of a coherently pumped polariton superfluid in the presence of defects. Here, in contrast to the corresponding equilibrium case, a weak residual drag force is always present even at extremely high polariton densities. ${ }^{4}$ Nevertheless, properties paradigmatic of equilibrium superfluids, such as frictionless flow of polariton bullets, ${ }^{5}$ quantized vortices, and metastable persistent flow ${ }^{6}$ and the appearance and disappearance of Cerenkov-like waves $^{7}$ have been recently observed in coherently driven exciton polaritons.

In view of the potential device applications, especially important is the unique versatility of the polaritonic system, which, combined with its high nonlinear properties, has been already demonstrated to produce parametric scattering ${ }^{8-10}$ and bistability. ${ }^{11}$ Here, the implementation of logic operations and gates comes in a natural way: by manipulating the nonlinear properties of the system using several lasers whose frequencies, angles of incidence, and intensities can be freely varied externally.

In this paper we investigate a new realization of a twocomponent polariton system coherently driven by two lasers with independently tunable frequencies, angles of incidence, and intensities. Firstly, we study the stability of the two polariton components when the two laser intensities are varied. Differently from the case of a single laser pump, where the system can only be bistable, we disclose a rich phase diagram where either one, two, or three stable states can coexist at given pumping conditions. Then we suggest possible easily tunable multistable hysteresis cycles when the two pumping lasers are varied up and down in intensities.

Alternatively, a multicomponent polariton fluid can be realized by considering the polarization degrees of freedom. Multistability of different polariton spin states has been recently proposed theoretically ${ }^{12}$ and confirmed experimentally ${ }^{13}$ by the observation of three stable spin states for a given excitation condition. For the case of two polarized components, multistability in space has also been theoretically proposed ${ }^{14,15}$ and experimentally observed. ${ }^{16}$ For the system with two pumping lasers presented here, the same kind of spatial multistability is expected but with much more complex features. Additionally, superimposed to the spatial multistability, interference fringes will appear due to the difference in frequency and momentum of the two pumping lasers. The analogy with the two-component polarized case suggests that aside from the interest in investigating multistability, two-component polariton condensates obtained with independent lasers can also be used to realize switches ${ }^{3}$ and memories.

The paper is organized as follows: in Sec. II, we present the model used to describe the steady-state behavior of polaritons excited by two continuous-wave lasers with different frequencies, wave vectors, and intensities. The results obtained within a linear-response framework are shown in Sec. III. In this section, we study the number of the possible solutions, their nature, and possible Kerr or parametric instabilities associated with them. As a consequence of the presence of multiple stable solutions, different cycles of hysteresis can be produced by varying, along different paths, the intensities of the two pumping lasers. Finally, Sec. IV contains the conclusions drawn from our analysis.

\section{MODEL}

The dynamics of resonantly driven microcavity polaritons ${ }^{8,17}$ can be described via a Gross-Pitaevskii 
equation for coupled cavity $\left(\psi_{C}\right)$ and exciton $\left(\psi_{X}\right)$ fields generalized to include decay and resonant pumping $(\hbar=1)$ :

$$
i \partial_{t}\left(\begin{array}{c}
\psi_{X} \\
\psi_{C}
\end{array}\right)=\left(\begin{array}{c}
0 \\
F
\end{array}\right)+\left[\hat{H}_{0}+\begin{array}{cc}
g_{X}\left|\psi_{X}\right|^{2} & 0 \\
0 & 0
\end{array}\right]\left(\begin{array}{l}
\psi_{X} \\
\psi_{C}
\end{array}\right) .
$$

The repulsive $\left(g_{X}>0\right)$ exciton-exciton interaction induces nonlinear dynamics of the eigenmodes of the single polariton Hamiltonian [lower and upper polariton, $\left.\omega_{\mathrm{LP}, \mathrm{UP}}(\mathbf{k})\right]$ :

$$
\hat{H}_{0}=\left(\begin{array}{cc}
\omega_{X}(-i \nabla)-i \kappa_{X} & \Omega_{R} / 2 \\
\Omega_{R} / 2 & \omega_{C}(-i \nabla)-i \kappa_{C}
\end{array}\right) .
$$

Here, we assume the cavity dispersion to be quadratic, $\omega_{C}(\mathbf{k})=\omega_{C}(0)+k^{2} /\left(2 m_{C}\right)$, with $m_{C}=2 \times 10^{-5} m_{0}$ (here, $m_{0}$ is the bare electron mass), we will neglect the exciton dispersion and consider the case of zero detuning at normal incidence, $\omega_{X}(\mathbf{k})=\omega_{X}(0)=\omega_{C}(0)$. The Rabi frequency, $\Omega_{R}=5.0 \mathrm{meV}$, and the excitonic and photonic decay rates, $\kappa_{X}=\kappa_{C}=0.05 \mathrm{meV}$ are chosen in the range of experimental values.

Because of the continuous decay, a stationary state requires a continuous injection of photons. Here, we consider two continuous-wave laser fields,

$$
F(\mathbf{r}, t)=F_{1} e^{i\left(\mathbf{k}_{1} \cdot \mathbf{r}-\omega_{1} t\right)}+F_{2} e^{i\left(\mathbf{k}_{2} \cdot \mathbf{r}-\omega_{2} t\right)},
$$

with independently tunable frequencies, $\omega_{1,2}$, and momenta, $\mathbf{k}_{1,2}$, which can be experimentally changed by changing the laser angle of incidence with respect to the growth direction.

We study the mean-field solutions of Eq. (1),

$$
\psi_{X, C}(\mathbf{r}, t)=\psi_{1_{X, C} s}^{s s} e^{i\left(\mathbf{k}_{1} \cdot \mathbf{r}-\omega_{1} t\right)}+\psi_{2_{X, C}^{s s}}^{s i\left(\mathbf{k}_{2} \cdot \mathbf{r}-\omega_{2} t\right)}
$$

and their stability with respect to small fluctuations within a linear-response analysis. Substituting the expression (4) into Eq. (1) we obtain four contributions, two of which oscillate at the main frequencies $\omega_{1}$ and $\omega_{2}$ and the additional two at the replica (or a satellite state) frequencies: $\omega_{1}-\Delta \omega$ and $\omega_{2}+$ $\Delta \omega$, where $\Delta \omega=\omega_{2}-\omega_{1}$. Similarly to what is done in the optical parametric oscillation (OPO) regime, ${ }^{18,19}$ where replica states in addition to the pump signal and idler states are neglected, here, we consider only the terms oscillating at the main frequencies $\omega_{1}$ and $\omega_{2}$. Later, see Eq. (6), we analyze the dynamical stability of the two-pump-frequency solution against the weak population of satellite states $\omega_{i} \pm \omega$ via parametric scattering processes. Through the paper, we will consider only dynamically stable two-pump-frequency solutions. In this approximation, we obtain the following mean-field equations for
$\psi_{1,2_{X, C} s s}:$

$$
\left\{\begin{array}{l}
{\left[\omega_{X}-\omega_{1}-i \kappa_{X}+G_{12}\right] \psi_{1_{X}}^{s s}+\frac{\Omega_{R}}{2} \psi_{1_{C}}^{s s}=0,} \\
{\left[\omega_{C}\left(\mathbf{k}_{1}\right)-\omega_{1}-i \kappa_{C}\right] \psi_{1_{C}}^{s s}+\frac{\Omega_{R}}{2} \psi_{1_{X}}^{s s}+F_{1}=0,} \\
{\left[\omega_{X}-\omega_{2}-i \kappa_{X}+G_{21}\right] \psi_{2_{X}}^{s s}+\frac{\Omega_{R}}{2} \psi_{2_{C}}^{s s}=0,} \\
{\left[\omega_{C}\left(\mathbf{k}_{2}\right)-\omega_{2}-i \kappa_{C}\right] \psi_{2_{C}}^{s s}+\frac{\Omega_{R}}{2} \psi_{2_{X}}^{s s}+F_{2}=0,}
\end{array}\right.
$$

where $G_{i j}=g_{X}\left(\left|\psi_{i_{X}}^{s s}\right|^{2}+2\left|\psi_{j_{X}}^{s s}\right|^{2}\right)$ with $i \neq j=1,2$. Note that the repulsive-interaction term between excitons in different states is two times larger the interaction term between excitons in the same mode, resulting in a nonuniform blue shift. The mean-field system of equations (5) can have up to nine solutions, i.e., six solutions more than in the case of one pumping laser, but, as discussed below, only a maximum of three solutions are stable.

The dynamical stability of the two-pump-frequency mean-field solution can be established by adding small fluctuations,

$$
\begin{aligned}
\psi_{X, C}(\mathbf{r}, t)= & e^{-i \omega_{1} t}\left[e^{i \mathbf{k}_{1} \cdot \mathbf{r}} \psi_{1_{X, C} s}^{s s}+\theta_{1_{X, C}}(\mathbf{r}, t)\right] \\
& +e^{-i \omega_{2} t}\left[e^{i \mathbf{k}_{2} \cdot \mathbf{r}} \psi_{2_{X, C}}^{s s}+\theta_{2_{X, C}}(\mathbf{r}, t)\right],
\end{aligned}
$$

where the fluctuation fields can be divided into particle-like and hole-like excitations, $\theta_{i_{X, C}}(\mathbf{r}, t)=\sum_{\mathbf{k}}\left[e^{-i \omega t+i \mathbf{k} \cdot \mathbf{r}} u_{i_{X, C} \mathbf{k}}+\right.$ $\left.e^{i \omega t+i\left(2 \mathbf{k}_{i}-\mathbf{k}\right) \cdot \mathbf{r}} v_{i_{X, C}}^{*}\right]$. Expanding Eq. (1) up to linear terms in $\theta_{1,2_{X, C}}$, we obtain four terms oscillating at frequencies $\omega_{1}-\Delta \omega \pm \omega$ and $\omega_{2}+\Delta \omega \pm \omega$, which we neglect, and four terms oscillating at $\omega_{i} \pm \omega$. In other words, we are checking the stability of our solution, where only the two states with frequencies $\omega_{1,2}$ are occupied, against the weak population of the satellite states $\omega_{i} \pm \omega$, which can be populated by parametric scattering processes. The fact that we consider only linear terms in $u_{i_{X, C} \mathbf{k}}$ and $v_{i_{X, C} \mathbf{k}}^{*}$ implies that we can obtain only the threshold conditions for such parametric processes, as well as the nature of the instability, whether of Kerr type or parametric type-see later. The equations for $u_{i_{X, C} \mathbf{k}}$ and $v_{i_{X, C} \mathbf{k}}^{*}$ can be written as an eigenvalue equation rearranging the excitations into an 8-component vector $\mathbb{U}^{\mathrm{T}}=\left(u_{1_{X}}, u_{1_{C}}, v_{1_{X}}, v_{1_{C}}, u_{2_{X}}, u_{2_{C}}\right.$, $\left.v_{2_{X}}, v_{2_{C}}\right)$ :

$$
\left[\omega \mathbb{I}-\left(\begin{array}{cc}
\mathbb{L}_{11 \mathbf{k}} & \mathbb{L}_{12 \mathbf{k}} \\
\mathbb{L}_{21 \mathbf{k}} & \mathbb{L}_{22 \mathbf{k}}
\end{array}\right)\right] \mathbb{U}_{\mathbf{k}}=0
$$

Here, matrices $\mathbb{L}_{i j \mathbf{k}}$ with $i \neq j$ are given by

$$
2 g_{X} e^{i\left(\mathbf{k}_{i}-\mathbf{k}_{j}\right) \cdot \mathbf{r}}\left(\begin{array}{lccc}
\psi_{i_{X}}^{s s} \psi_{j_{X}}^{s s \star} & 0 & \psi_{i_{X}}^{s s} \psi_{j_{X}}^{s s} & 0 \\
0 & 0 & 0 & 0 \\
-\psi_{i_{X}}^{s s \star} \psi_{j_{X}}^{s s \star} & 0 & -\psi_{i_{X}}^{s s \star} \psi_{j_{X}}^{s s} & 0 \\
0 & 0 & 0 & 0
\end{array}\right)
$$


and $\mathbb{L}_{j j \mathbf{k}}$ are given by

$$
\left(\begin{array}{lccc}
\omega_{X}-\omega_{j}-i \kappa_{X}+g_{X}\left|\psi_{X}^{s s}\right|^{2} & \frac{\Omega_{R}}{2} & g_{X} \psi_{j_{X}}^{s s} \psi_{j_{X}}^{s s} & 0 \\
\frac{\Omega_{R}}{2} & \omega_{C}(\mathbf{k})-\omega_{j}-i \kappa_{C} & 0 & 0 \\
-g_{X} \psi_{j_{X}}^{s s \star} \psi_{j_{X}}^{s s \star} & 0 & -\omega_{X}\left(2 \mathbf{k}_{j}-\mathbf{k}\right)+\omega_{j}-i \kappa_{X}-g_{X}\left|\psi_{X}^{s s}\right|^{2} & -\frac{\Omega_{R}}{2} \\
0 & 0 & -\frac{\Omega_{R}}{2} & -\omega_{C}\left(2 \mathbf{k}_{j}-\mathbf{k}\right)+\omega_{j}-i \kappa_{C}
\end{array}\right),
$$

with $\left|\psi_{X}^{s s}\right|^{2}=2\left(\left|\psi_{1_{X}}^{s s}\right|^{2}+\left|\psi_{2_{X}}^{s s}\right|^{2}\right)$ being the total excitonic density. At given values of the pumping strength $F_{1}$ and $F_{2}$, the solutions of the mean-field equations (5) are stable if all the eight eigenvalues, $\left[\mathrm{LP}_{j}^{ \pm}(\mathbf{k}), \mathrm{UP}_{j}^{ \pm}(\mathbf{k})\right]$, of Eq. (7) have negative imaginary part for every value of the momentum $\mathbf{k}$.

\section{RESULTS}

For some choices of the system parameters, we find that the number of stable solutions can be larger than one. In the case of one pumping laser, the typical $S$-like shape dependence of the polariton-field intensity on the pump strength, also referred to as optical bistability, can be explained in terms of the nonlinear blue shift induced by the polariton-polariton interaction. ${ }^{18,20}$ When the laser frequency is well above the bare lower polariton dispersion, $\omega_{p}>\omega_{\mathrm{LP}}\left(\mathbf{k}_{p}\right)$, and the pump intensity increases from low values, the polariton population remains small because it is hard for the laser to inject polartions with a different energy. However, increasing the pump power, the blue shift pulls the polariton energy toward resonance with the pump, causing the population to grow superlinearly and eventually to abruptly jump to a high value when the pump intensity reaches a critical value $I_{1}$. In the opposite situation, when the laser intensity is decreased from high values, the polariton energy is blue detuned close to the pumping laser frequency and, therefore, the cavity is efficiently filled by the laser even at low pumping intensities. In this case the polariton population jumps down back at low polariton densities for a value $I_{2}$ of the pump strength lower than $I_{1}$. The two jumps at different values of the pump intensity cause therefore a hysteresis cycle. As explained below, in the case of two-component fluids, the situation becomes even richer.

We fix both laser frequencies to be blue detuned with respect to the bare polariton dispersion: $\omega_{1,2}=\omega_{\mathrm{LP}}\left(\mathbf{k}_{1,2}\right)+0.3 \mathrm{meV}$, with $\mathbf{k}_{1}=0.25 \mu \mathrm{m}^{-1}$ and $\mathbf{k}_{2}=0.7 \mu \mathrm{m}^{-1}$. We plot in panel I of Fig. 1 the phase diagram showing the regions with a different number of stable solutions (either one, two, or three) as a function of the two rescaled pumping intensities $F_{1,2}^{\prime}=$ $\sqrt{g_{x}} F_{1,2} \mathrm{meV}^{3 / 2}$. In order to understand better the structure of this phase diagram, we show in Fig. 2 the total exciton density, $g_{X}\left|\psi_{X}^{s s}\right|^{2}$, when the pump intensity $F_{2}^{\prime}$ is kept constant at different values and $F_{1}^{\prime}$ is varied. When the constant pump $F_{2}^{\prime}$ has a small value (see Fig. 2 top left panel), the dependence of the population on the varying pump intensity $F_{1}^{\prime}$ is similar to the one-fluid case showing bistability with an $S$-like shape. For higher values of the constant pump $F_{2}^{\prime}$ (see Fig. 2 lower left panel), the number of possible solutions increases but just two are found to be stable. Finally, when $F_{2}^{\prime}$ is further increased, the set of possible solutions further goes up but only a maximum of three are found to be stable.
The coexistence of three solutions, corresponding to the black regions of Fig. 1, can be understood as follows: when the two pump intensities increase from low values, the polariton population is small because its energy is far below the laser frequencies, resulting in one stable solution. In the opposite
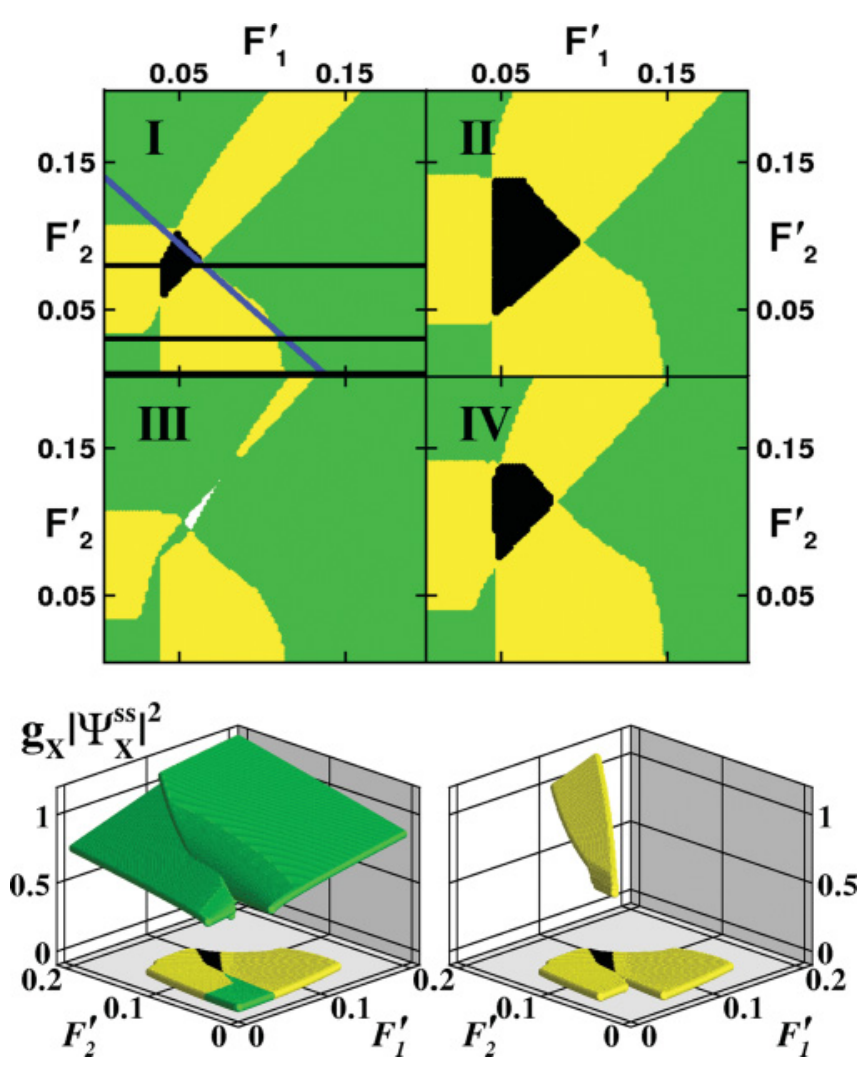

FIG. 1. (Color online) 2D panels: phase diagram showing the number of stable solutions as a function of the rescaled pump intensities $F_{1,2}^{\prime}=\sqrt{g_{x}} F_{1,2} \mathrm{meV}^{3 / 2}$. White, green, yellow, and black regions correspond to zero, one, two, or three stable solutions, respectively. In panels I and II, $\mathbf{k}_{1}=0.25 \mu \mathrm{m}^{-1}$ and $\mathbf{k}_{2}=0.7 \mu \mathrm{m}^{-1}$ while in panels III and IV, $\mathbf{k}_{1}=0.0 \mu \mathrm{m}^{-1}$ and $\mathbf{k}_{2}=0.7 \mu \mathrm{m}^{-1}$. In the left panels (I and III), $\omega_{1,2}=\omega_{\mathrm{LP}}\left(\mathbf{k}_{1,2}\right)+0.3 \mathrm{meV}$, while in the right panels (II and IV), $\omega_{1,2}=\omega_{\mathrm{LP}}\left(\mathbf{k}_{1,2}\right)+0.4 \mathrm{meV}$. The horizontal black line lies at the three fixed values of $F_{2}^{\prime}$ corresponding to the three panels of Fig. 2, while the blue diagonal line is the path used to plot Fig. 5. 3D panels: plots of $g_{X}\left|\psi_{X}^{s s}\right|^{2}$ in meV as a function of $F_{1,2}^{\prime}$ with parameters equal to panel I. Stable solutions with higher populations are shown in green, stable solution with the second higher population in yellow, and third stable solution with lower population in black. All the solutions are shown in the left panel. Since the upper green branches hide a yellow upper branch, the right panel shows only the yellow and black solutions. 


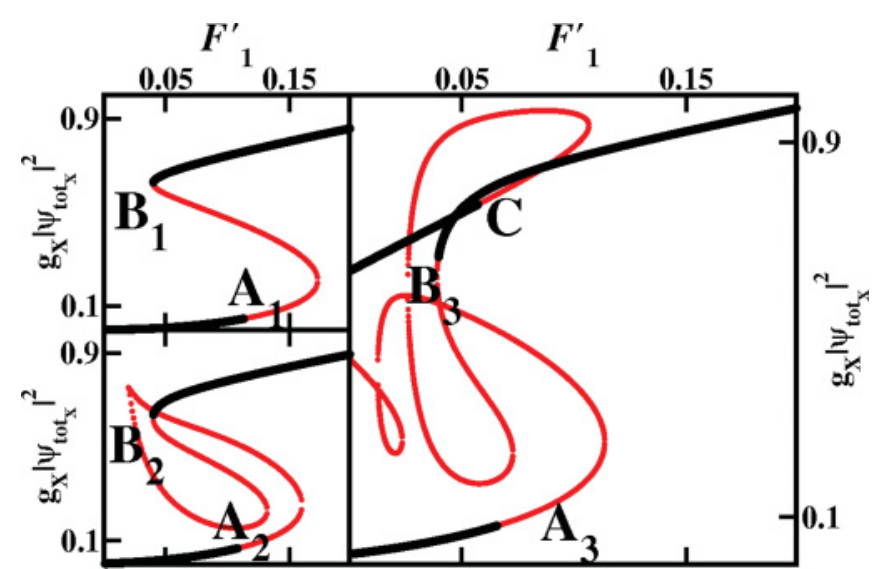

FIG. 2. (Color online) Stability curves of the total exciton density $g_{X}\left|\psi_{X}^{s s}\right|^{2}$ in meV (red dotted curves represent unstable solutions, black lines are stable solutions) for fixed pump intensities as a function of $F_{1}^{\prime}$ for $F_{2}^{\prime}=0.00001 \mathrm{meV}^{3 / 2}$ (top left), $F_{2}^{\prime}=0.025 \mathrm{meV}^{3 / 2}$ (bottom left), and $F_{2}^{\prime}=0.08 \mathrm{meV}^{3 / 2}$ (right). Points $A_{i}\left(B_{i}\right), i=1,2,3$ correspond to the cases when the lower (upper) branch of the stability curve becomes unstable (see Figs. 3 and 4). Point $C$ does not have a counterpart in the one-fluid case and corresponds to the cases when the second high branch of the stability curve becomes unstable (see Fig. 4).

situation, when the intensity of one of the lasers decreases from high values, the polariton population is high and its dispersion is significantly blue detuned with respect to the bare one. Such a blue shift can be sustained by any of the two lasers, thus giving two additional stable solutions for the same values of the pump intensities. Therefore a maximum of three stable solutions can be expected. This is also evident while considering the partial densities for particular values of the pump strength at which three stable solutions are present (black region of Fig. 1), e.g., $F_{1}^{\prime} \equiv \sqrt{g_{X}} F_{1}=0.05 \mathrm{meV}^{3 / 2}$ and $F_{2}^{\prime} \equiv \sqrt{g_{X}} F_{1}=0.08 \mathrm{meV}^{3 / 2}$. Here, the solution with lower total polariton density corresponds to partial densities $g_{X}\left|\psi_{1_{X}}^{s s}\right|^{2}=0.009 \mathrm{meV}$ and $g_{X}\left|\psi_{2_{X}}^{s s}\right|^{2}=0.023 \mathrm{meV}$. The other two solutions correspond to a high value of just one of the two partial populations: $g_{X}\left|\psi_{1_{X}}^{s s}\right|^{2}=0.008 \mathrm{meV}$ and $g_{X}\left|\psi_{2_{X}}^{s s}\right|^{2}=$ $0.609 \mathrm{meV}$ in one case and $g_{X}\left|\psi_{1_{X}}^{s s}\right|^{2}=0.646 \mathrm{meV}$ and $g_{X}\left|\psi_{2 X}^{s s}\right|^{2}=0.010 \mathrm{meV}$ in the other. Note that this situation is similar to the case of two-component condensates obtained with two spins. However, while in the spin-dependent case the two lasers pump the two spin populations with different intensities but at the same angle and energy, here the two pumps are independent also in angle and in energy. This analogy is also visible in Eq. (5). However, the difference between our system of equations and the spin-dependent case is that here the interaction between different components is twice the interaction between particles in the same component.

In panel II of Fig. 1 we plot the phase diagram for the same parameters as in panel I but with the two pumping lasers $0.4 \mathrm{meV}$ blue detuned with respect to the bare lower polarization (LP) branch. We see that the effect of the increased detuning is simply to stretch the phase diagram. Since the two pumps are further apart from the LP branch, it is more difficult to inject polaritons into the cavity, and thus the need for higher pump intensities. In panel IV of Fig. 1 we show that a similar phase diagram can be obtained by changing the $\mathbf{k}$ vector of pump 1 from 0.25 to $0.0 \mu \mathrm{m}^{-1}$. We observe that the multistability is quite robust with respect to the choice of the parameters and, therefore, it should be within an experimental reach. An interesting configuration is plotted in panel III of the same figure. Here, a region with no stable solutions appears in the central part of the plot (white region). The instability of this region can be understood by noting that with a pump at $\mathbf{k}=0.0$ and just slightly blue detuned from the LP and a pump at $\mathbf{k}=0.7$, close to the inflection point of the LP, it is easy to satisfy phase-matching conditions for parametric-scattering processes. For this set of parameters the system is in a configuration unstable to the population of satellite states by scattering processes. For the other three sets of parameters, shown in the remaining three panels of Fig. 1, it is also possible to find regions of the phase diagram where no solutions are stable. These are the regions where the proposed solution, given by Eq. (4), where only the two frequency states, $\omega_{1}$ and $\omega_{2}$, are populated, is not a stable solution because satellite states start also to be populated-our analysis is giving the threshold for this to happen.

To further discuss the stability of the system with respect to small perturbations, we plot the dispersion of the imaginary part of the excitation eigenfrequency $\omega=\mathrm{LP}_{j}^{ \pm}$for several points of the stability curves shown in Fig. 2. We start with the cases where the lower branch of the stability curve became unstable at points $A_{i}$. For very small values of $F_{2}^{\prime}$ the imaginary part of the dispersion (see top panel of Fig. 3) shows two peaks for given values of $\mathbf{k}$. One peak lies at higher value $\mathbf{k}_{+}=0.83 \mu \mathrm{m}^{-1}$ and one peak at lower value $\mathbf{k}_{-}=$ $-0.33 \mu \mathrm{m}^{-1}$ with $\mathbf{k}_{+}+\mathbf{k}_{-}=2 \mathbf{k}_{1}$. This two-peak structure is a precursor of a parametric instability due to the scattering between two particles in the component of the condensate with momentum $\mathbf{k}_{1}$. This situation corresponds exactly to the case of one-component fluids. When the pump intensity $F_{2}^{\prime}$ is slightly increased (see middle panel), we observe six other peaks appearing in the imaginary part of the dispersion. Two of these new peaks are such that $\mathbf{k}_{+}+\mathbf{k}_{-}=2 \mathbf{k}_{1}$ (blue lines) while the other four can be combined to identify two different scattering processes with $\mathbf{k}_{+}+\mathbf{k}_{-}=2 \mathbf{k}_{2}$ (red curves). This more complicated structure of the imaginary parts of the eigenvalues is consistent with the fact that with two components richer mechanisms of scattering might occur. When the intensity of $F_{2}^{\prime}$ is further increased (see lower panel), still four different scatterings may occur but, in this case, it is the scattering between two particles with $\mathbf{k}_{2}$ that induces the instability of the system.

For the transition from stable to unstable regions of the higher branch of the stability curve we plot the dispersion of the imaginary part of the excitation eigenfrequency $\omega=\mathrm{LP}_{j}^{ \pm}$ for points $\mathrm{B}_{i}$ in Fig. 4. In analogy with the case of fluids with one component, for low intensities of pump 2, the imaginary part of the dispersion shows a peak at the wave vector of pump 1 , a clear precursor of a Kerr instability. When the intensity of pump 2 is increased, new peaks appear in the imaginary part. In the case of point $\mathrm{B}_{2}$, two new peaks are precursors of a parametric instability for the state with $\mathbf{k}=0.25 \mu \mathrm{m}^{-1}$ even if the mechanism responsible for the instability of the solution is still of Kerr type. It is only when the pump intensity $F_{2}^{\prime}$ is further increased (point $\mathrm{B}_{3}$ ) that the two peaks at $k_{-}=0.15$ 


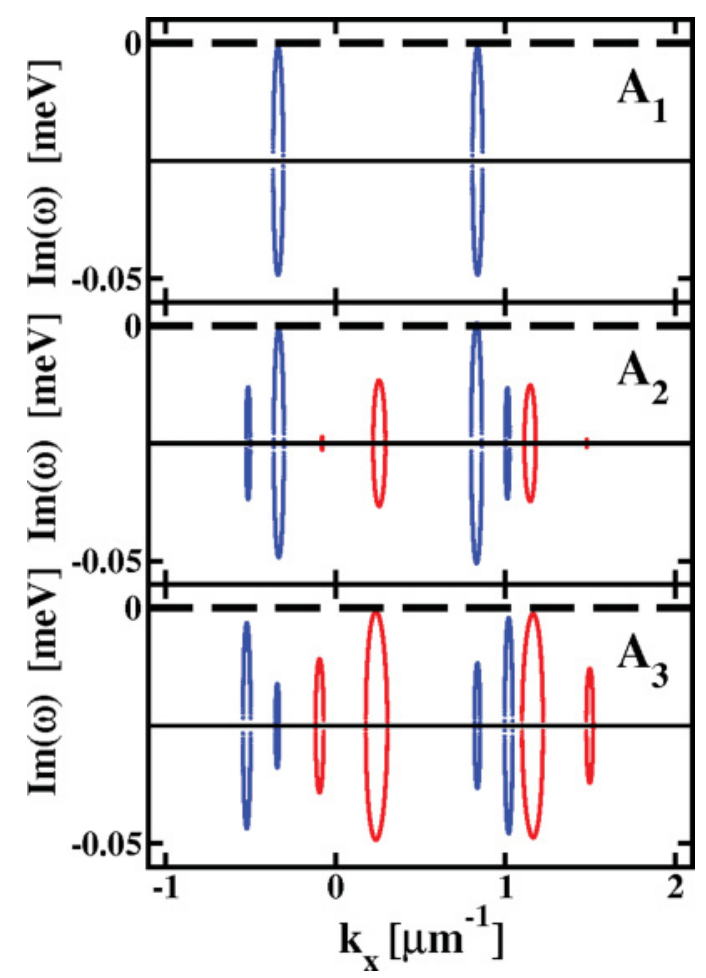

FIG. 3. (Color online) Dispersion of the imaginary part of the excitation eigenfrequency $\omega=\mathrm{LP}_{j}^{ \pm}$. The three panels correspond to points $A_{i}$ with $i=1,2$, and 3 of Fig. 2, where the lower part of the stability curves become unstable. In blue (red) the parts corresponding to the scattering of two particles with $\mathbf{k}=\mathbf{k}_{1}\left(\mathbf{k}=\mathbf{k}_{2}\right)$.

and $k_{+}=0.35 \mu \mathrm{m}^{-1}$ became more important and the mechanism of instability is of the parametric type. Finally, in the lower right panel (corresponding to point C) of Fig. 4 a single peak at $\mathbf{k}=\mathbf{k}_{1}$ is the precursor of a Kerr-type instability that ends the region with three stable solutions in the right panel of Fig. 2.

Multistability also manifests itself in a hysteresis loop for the populations and emission intensities obtained with

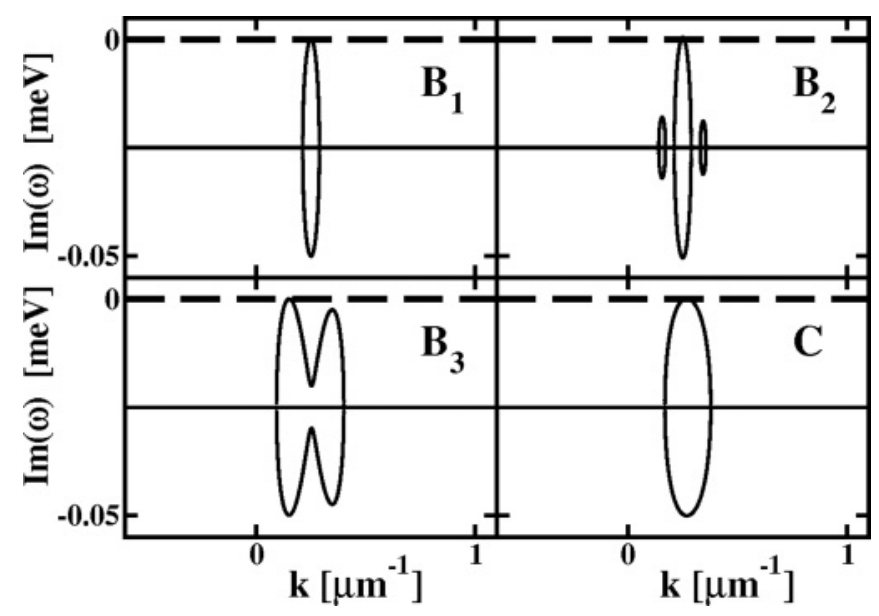

FIG. 4. Dispersion of the imaginary part of the excitation eigenfrequency $\omega=\mathrm{LP}_{j}^{ \pm}$. The three panels correspond to points $\mathrm{B}_{i}$ with $i=1,2,3$ and $\mathrm{C}$ of Fig. 2, where the higher part of the stability curve becomes unstable.

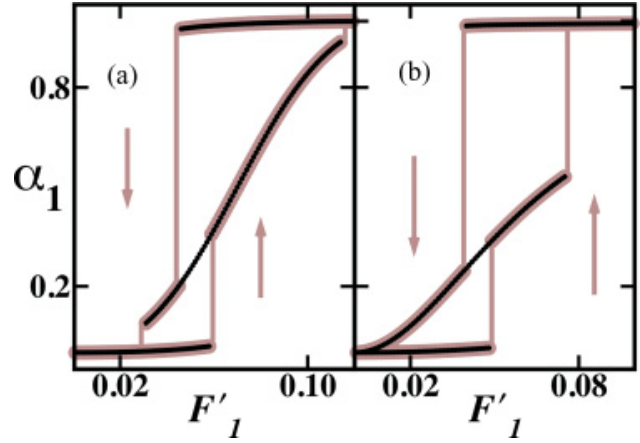

FIG. 5. (Color online) Hysteresis cycles of $\alpha_{1}=\left|\psi_{1_{X}}^{s s}\right|^{2} /$ $\left(\left|\psi_{1_{X}}^{s s}\right|^{2}+\left|\psi_{2_{X}}^{s s}\right|^{2}\right)$ (dimensionless) as a function of $F_{1}^{\prime}$ for different values of $F_{2}^{\prime}$-stable solutions are in black, while the hysteresis cycle performed by the system is in brown. (a): $F_{2}^{\prime}=0.14-F_{1}^{\prime}$ as in the blue line of Fig. 1. (b): $F_{2}^{\prime}=0.08 \mathrm{meV}^{3 / 2}$ as in the horizontal black line of Fig. 1.

a cycle of first increasing and later decreasing the pumping intensities. Here, the presence of three stable solutions gives more complicated loops than the ones obtained for a bistable system in a single-component polariton fluid. In order to study this aspect, we calculate the exciton emission intensity at a given frequency $\omega_{i}$ normalized to the total exciton emission intensity, $\alpha_{i}=\left|\psi_{i_{X}}^{s s}\right|^{2} /\left(\left|\psi_{1_{X}}^{s s}\right|^{2}+\left|\psi_{2_{X}}^{s s}\right|^{2}\right)$, along a closed path of varying pumping intensities. The panels (a) and (b) of Fig. 5 respectively show the hysteresis cycles of $\alpha_{1}$ when the two pump intensities change along either the blue or the higher horizontal black line of Fig. 1. In panel (a), one starts from a low value of $F_{1}^{\prime}$ taking $F_{2}^{\prime}=0.13 \mathrm{meV}^{3 / 2}$ so that the population of state 2 is much higher than the population of state 1 , i.e., $\alpha_{1} \ll 1$. Increasing $F_{1}^{\prime}$, the two populations smoothly evolve until $F_{1}^{\prime} \approx 0.05 \mathrm{meV}^{3 / 2}$ and $F_{2}^{\prime} \approx 0.09 \mathrm{meV}^{3 / 2}$. At this point $F_{2}^{\prime}$ is too weak to sustain high population densities in state 2 and, therefore, the system jumps to a new stable configuration, in which the populations of both states are low, i.e., $\alpha_{1} \approx 0.5$. A further increase of $F_{1}^{\prime}$ produces a smooth evolution of the two populations until $F_{1}^{\prime} \approx 0.11 \mathrm{meV}^{3 / 2}$ when the system jumps to a third configuration with a population in state 1 much higher than in state 2 , i.e., $\alpha_{1} \approx 1$. When we revert the variation of the pumping intensities along the same path, the jumps to states corresponding to intermediate and low values of $\alpha_{1}$ are shifted to the left of the ones just described for increasing $F_{1}^{\prime}$. The multistable hysteresis loop shown in Fig. 5(a) is related to the fact that the two pumping lasers are at different pumping angles, $\mathbf{k}_{i}$, and pumping frequencies, $\omega_{i}$. Therefore, the jumps from low to high population for each component appear at different values of the pumping intensities, producing the multistable behavior of $\alpha_{1}$.

A similar situation occurs when the system evolves along a path on which one of the pumping intensities remains constant while the other varies (black horizontal line at $F_{2}^{\prime}=0.08$ of panel 1 in Fig 1, as shown in Fig. 5(b). Starting with $F_{1}=0$, $\alpha_{1}$ increases smoothly from zero, following the lower branch up to $F_{1}^{\prime}=0.06 \mathrm{meV}^{3 / 2}$. At this point $\alpha_{1}$ jumps from values of the order of 0.01 to 0.3 , corresponding to a population of state 1 being smaller but non-negligible compared to state 2 . As $F_{1}^{\prime}$ further increases up to $0.08 \mathrm{meV}^{3 / 2}, \alpha_{1}$ again jumps abruptly to values of the order of 0.95 . In the reverse process, $F_{1}^{\prime}$ is 
decreased completing the loop. Also in this case, the jumps from high to low values of $\alpha_{1}$ are shifted to the left because of the different angles and energies at which the lasers are pumping the two components of the fluid. It is worth noting that the length and the height of the different plateaus of the hysteresis loops can be efficiently tuned by carefully choosing the path followed by the intensities, angles, and frequencies of the pumping lasers.

\section{CONCLUSIONS}

To summarize, we have studied the stability of a twocomponent exciton-polariton fluid under resonant excitation of two pumping lasers with independently tunable frequencies, angles of incidence, and intensities. We have studied the effect of the detuning between the laser pump and the bare LP branch, and discussed the different kinds of instability that might occur for different values of the parameters. We have shown that, even though the kind of instabilities is the same as in the one-component case (Kerr or parametric), here the interplay between different instabilities in the two components of the fluid can produce a much richer picture. Moreover, we have shown that since each component of the fluid jumps between stable states of its population at different values of the pumping intensity, the system sustains multistable hysteresis loops that can be easily modulated by changing the parameters of the pumping lasers. Finally, we believe that, due to the wide range of parameters for which the system is multistable, and due to the increased number of degrees of freedom with respect to the spin-dependent case, multistability and hysteresis loops should be within an experimental reach. Therefore, the novel system of a two-component fluid is a promising candidate for the realization of optical switches and memories.

\section{ACKNOWLEDGMENTS}

We thank I. Carusotto and D. Whittaker for discussions. This research has been supported by the Spanish MEC (MAT2008-01555, QOIT-CSD2006-00019) and CAM (S-2009/ESP-1503). F.M.M. acknowledges financial support from the program Ramón y Cajal. *emiliano.cancellieri@uam.es

${ }^{1}$ J. Keeling and N. G. Berloff, Nature (London) 457, 273 (2009).

${ }^{2}$ V. M. Menon et al., Nat. Photon. 4, 345 (2010).

${ }^{3}$ A. Amo et al., Nat. Photon. 4, 361 (2010).

${ }^{4}$ E. Cancellieri, F. M. Marchetti, M. H. Szymanska, and C. Tejedor, Phys. Rev. B 82, 224512 (2010).

${ }^{5}$ A. Amo et al., Nature (London) 457, 291 (2009).

${ }^{6}$ D. Sanvitto et al., Nat. Phys. 6, 527 (2010).

${ }^{7}$ A. Amo et al., Nat. Phys. 5, 805 (2009).

${ }^{8}$ I. Carusotto and C. Ciuti, Phys. Rev. Lett. 93, 166401 (2004).

${ }^{9}$ P. G. Savvidis et al., Phys. Rev. Lett. 84, 1547 (2000).

${ }^{10}$ R. M. Stevenson, V. N. Astratov, M. S. Skolnick, D. M. Whittaker, M. Emam-Ismail, A. I. Tartakovskii, P. G. Savvidis, J. J. Baumberg, and J. S. Roberts, Phys. Rev. Lett. 85, 3680 (2000).

${ }^{11}$ A. Baas, J. P. Karr, M. Romanelli, A. Bramati, and E. Giacobino, Phys. Rev. B 70, 161307 (2004)
${ }^{12}$ N. A. Gippius, I. A. Shelykh, D. D. Solnyshkov, S. S. Gavrilov, Y. G. Rubo, A. V. Kavokin, S. G. Tikhodeev, and G. Malpuech, Phys. Rev. Lett. 98, 236401 (2007).

${ }^{13}$ T. K. Paraïso et al., Nat. Mater. 9, 655 (2010).

${ }^{14}$ I. A. Shelykh, Y. G. Rubo, G. Malpuech, D. D. Solnyshkov, and A. Kavokin, Phys. Rev. Lett. 97, 066402 (2006).

${ }^{15}$ I. A. Shelykh, T. C. H. Liew, and A. V. Kavokin, Phys. Rev. Lett. 100, 116401 (2008).

${ }^{16}$ C. Adrados, A. Amo, T. C. H. Liew, R. Hivet, R. Houdre, E. Giacobino, A. V. Kavokin, and A. Bramati, Phys. Rev. Lett. 105, 216403 (2010).

${ }^{17}$ C. Ciuti, P. Schwendimann, and A. Quattropani, Semicond. Sci. Technol. 18, S279 (2003).

${ }^{18}$ D. M. Whittaker, Phys. Rev. B 71, 115301 (2005).

${ }^{19}$ M. Wouters and I. Carusotto, Phys. Rev. Lett. 99, 140402 (2007).

${ }^{20}$ C. Ciuti and I. Carusotto, Phys. Status Solidi B 242, 2224 (2005). 